\title{
Témoignages croisés sur les constructions antiques en terre crue : textes latins et données archéologiques
}

Intersecting evidence on ancient mud constructions: latin literature and archaeological data

Testimonios cruzados sobre las construcciones en tierra cruda de la Antigüedad. Textos en latín y datos arqueológicos

Claire-Anne de Chazelles

\section{OpenEdition}

Journals

Édition électronique

URL : https://journals.openedition.org/tc/55

DOI : $10.4000 /$ tc. 55

ISSN : 1952-420X

Éditeur

Éditions de l'EHESS

Édition imprimée

Date de publication : 1 décembre 2003

Pagination : 1-27

ISSN : 0248-6016

Référence électronique

Claire-Anne de Chazelles, «Témoignages croisés sur les constructions antiques en terre crue : textes latins et données archéologiques », Techniques \& Culture [En ligne], 41 | 2003, mis en ligne le 13 janvier 2005, consulté le 29 septembre 2022. URL : http://journals.openedition.org/tc/55 ; DOI : https:// doi.org/10.4000/tc.55

Ce document a été généré automatiquement le 29 septembre 2022.

Tous droits réservés 


\title{
Témoignages croisés sur les constructions antiques en terre crue : textes latins et données archéologiques
}

\author{
Intersecting evidence on ancient mud constructions: latin literature and \\ archaeological data
}

Testimonios cruzados sobre las construcciones en tierra cruda de la Antigüedad. Textos en latín y datos arqueológicos

\section{Claire-Anne de Chazelles}

\section{NOTE DE L'ÉDITEUR}

La traduction des textes latins est de Sabine ALBERT (Éducation Nationale).

1 Les textes antiques -et particulièrement les textes latins - qui traitent directement ou indirectement de l'architecture domestique sont peu nombreux et tous bien connus. L'ouvrage de Vitruve mis à part, il s'agit le plus souvent de courtes mentions : conseils pratiques donnés par les « agronomes " pour la construction de bâtiments liés à la vie rurale, anecdotes de voyages, ou encore, mais plus rarement, allusions dans la littérature. Sur le thème particulier des constructions en terre crue, le recensement des références a déjà été fait et le propos de cet article n'est pas d'en reproduire une liste exhaustive (Lequément 1985). Néanmoins, comme le sens de ces citations demeure souvent imprécis ou sujet à des interprétations ambiguës, la mise en parallèle des informations qu'elles restituent avec les données archéologiques recueillies au cours des deux dernières décennies constitue un exercice intéressant. 
2 En effet, des progrès considérables ont été accomplis en archéologie depuis le début des années 1980, période qui coïncidait avec la découverte d'un immense patrimoine en terre crue jusqu'alors ignoré2.

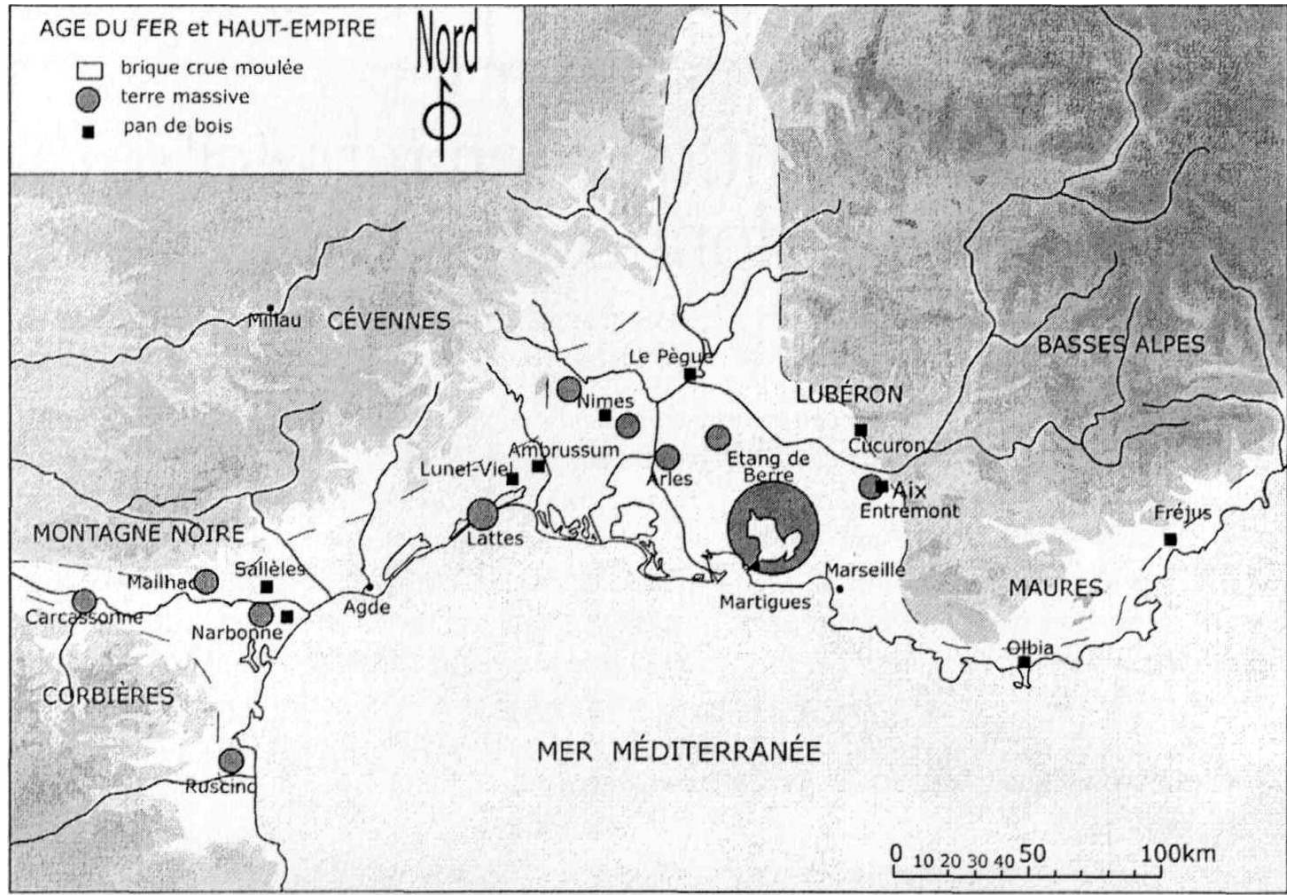

Carte des sites archéologiques en France

Actuellement, nous sommes capables non seulement de reconnaître les constructions en terre préhistoriques, proto-historiques, gallo-romaines et médiévales, mais également d'en identifier les modes de mise en œuvre avec une large gamme de nuances. Nous parvenons à ébaucher une histoire de ces techniques sur une très longue durée, à comparer les régions entre elles, ainsi que les périodes, et nous pouvons pointer l'apparition de tel ou tel procédé ou la disparition de tel autre.

Parmi les auteurs latins qui mentionnent ou décrivent des structures en terre, plusieurs sont ces fameux "agronomes" dont les préoccupations concernent aussi bien l'architecture des maisons et des bâtiments agricoles que l'agronomie elle-même: Varron (II s. av. n.è.), Caton ( $\mathrm{I}^{\mathrm{er}} \mathrm{s}$. av. n.è.), puis Columelle ( $\mathrm{I}^{\mathrm{er}} \mathrm{s}$. de n.è.) et Palladius (IV s. de n.è.), les deux derniers ayant essentiellement repris et commenté les textes antérieurs. On doit à l'architecte Vitruve, qui vécut sous le règne d'Auguste, une somme d'informations capitales sur les matériaux et leur préparation, ainsi que sur différents procédés constructifs dont il a comparé les avantages. En marge des aspects techniques, son ouvrage comporte quelques observations ethnographiques sur l'habitat romain et sur celui des provinces voisines de l'Italie : Gaules, Ibérie et Lusitanie. L'Histoire naturelle de Pline ( $\mathrm{I}^{\mathrm{er}} \mathrm{s}$. de $n$. è.), qui recèle des passages directement copiés chez Vitruve, offre également des descriptions originales du plus grand intérêt.

Par ailleurs, si on peut glaner dans des récits de voyageurs (en particulier Strabon ${ }^{3}$ ) ou au fil des ouvrages d'histoire quelques évocations de constructions en terre, elles attirent souvent l'attention sur la vulnérabilité ${ }^{4}$ ou sur l'aspect misérable ${ }^{5}$ de cette architecture.

6 Les textes réunis ici donnent une idée assez précise des savoir-faire empiriques dont disposaient maçons et architectes romains et montrent, en particulier avec Vitruve, le 
souci précoce de rationaliser et de normaliser des procédés, sans doute utilisés spontanément par les auto-constructeurs, afin de les hisser au rang de techniques savantes. Les descriptions de Vitruve, tout comme les remarques de Varron ou de Pline sur quelques particularismes régionaux, permettent de déterminer ce qui relève de la pratique des maçons romains et ce qui semble propre à d'autres cultures.

7 Bien entendu, il n'est pas question de confronter directement les textes latins et les données de fouilles, d'abord en raison de la distorsion chronologique -les vestiges archéologiques auxquels on se réfère étant souvent antérieurs à la date d'écriture des textes- et, ensuite, parce que ces données proviennent essentiellement de la France méridionale et du nord-est de l'Espagne et non de la péninsule italique. Pour autant, loin d'être antinomiques, les informations se rejoignent et se complètent mutuellement sur la plupart des thèmes. J'ai choisi de mettre l'accent sur la construction des murs car ce sujet offre la plus grande complexité : brique crue, bauge, pisé, torchis et pans de bois sont donc examinés selon des points de vue technique et ethno-historique.

8 Les textes relatifs aux finitions et aux toitures sont trop succincts et trop rares pour donner lieu à une véritable discussion et les écrits littéraires n'ont pas été pris en compte.

\section{La brique crue} pierres, comme dans le territoire de Tusculum, en briques cuites comme dans le territoire gaulois, en briques crues, comme dans la province de Sabine, en un mélange de terre et de gravier aggloméré dans des moules, comme en Espagne et dans le territoire de Tarente ${ }^{7}$. trouvent dans la construction des bâtiments grecs: l'un est dit pentadoron, l'autre tetradoron.

- Caton, De agricultura, XVII, 14, 4 dessus du niveau du sol, le reste des murs en briques crues $^{6}$.

- Varron, Res rusticae, I, 14, 4

- Vitruve, De architectura, II, 3

(À propos des briques crues) En effet, il ne faut pas les faire de terre mêlée de sable, ni de gravier, ni de sabulo, car si elles sont faites avec ces matériaux, d'abord les briques sont trop lourdes, ensuite, lorsque la pluie les éclabousse, elles tombent en morceaux et se désagrègent et les pailles n'y adhèrent pas. Au contraire, il faut les faire à l'aide de terre argileuse blanchâtre, ou avec de la terre rouge (ocre ?) ou du sabulo mâles.

On fait trois types de briques : l'un d'eux qui est appelé « lydien » en grec, est celui que nous utilisons, il est long d'un pied et demi et large d'un pied. Les deux autres types se

En même temps que ces briques, on fait des demi-briques. Quand on bâtit, on dispose d'un côté une rangée de briques et, de l'autre côté, des demi-briques.

- Vitruve, De architectura, II, 8

[...] Mais les murs de briques, s'ils ne sont pas faits de deux ou trois briques et n'ont qu'un pied et demi d'épaisseur, ne peuvent soutenir plus d'un étage ${ }^{10}$. 
19 [...] Sur le haut des murs, au-dessous du toit, il faut établir une maçonnerie de terre cuite d'environ un pied et demi de hauteur, formant une corniche en surplomb ${ }^{11}$.

- Pline, Naturae historiarum, XXXV, 49

Les briques ne doivent pas être faites avec un matériau sableux à gros grains ou à grains fins, encore moins caillouteux, mais d'une terre argileuse blanche ou de terre rouge, ou même de sabulo, si c'est du «sabulo mâle ». [...] il faut que le mélange ait bien macéré avant d'être façonné.

[...] Il y en a trois sortes : la Lydienne, que nous utilisons, est longue d'un pied et demi et large d'un pied $[. . .]^{12}$.

— Pline, Naturae historiarum, VII, 57, 194

Les premiers à construire des maisons en briques crues furent les frères Euryalos et Hyperbius, d'Athènes [...] $]^{13}$.

25 - Palladius, Opus agriculturae, I, XI

Parce que si vous voulez faire des murs de briques dans la maison du propriétaire, vous devez procéder de sorte que sur la partie supérieure des murs achevés, on place sous le toit une maçonnerie en terre cuite, haute d'un pied et demi, avec des corniches saillantes $[\ldots]^{14}$.

27 - Palladius, Opus agriculturae, VI, 12

Mais que les côtés soient d'une longueur de deux pieds, d'une largeur d'un pied et d'une hauteur de 4 pouces ${ }^{15}$.

29 La portée de ces textes est très inégale : simples témoignages sur l'emploi de la brique crue au cours des deux siècles précédant le règne d'Auguste, dans les cas de Caton et de Varron, mais véritables recommandations techniques en ce qui concerne les trois autres. Toutefois, les similitudes flagrantes entre les textes trahissent le recopiage pur et simple de l'ouvrage original de Vitruve.

Si l'on se fie à ce dernier, durant la période augustéenne, la brique usuelle des Romains mesurait un pied et demi de long sur un pied de large, alors qu'elle atteindrait deux pieds de longueur quelques siècles plus tard, aux dires de Palladius. Des variations de modules aussi importantes peuvent s'expliquer par un accroissement de la taille des briques au Bas-Empire, bien que le format démesuré indiqué par Palladius implique un poids élevé et une manipulation difficile ${ }^{16}$. Pourtant, si l'on se réfère aux données de l'archéologie, aucune préférence pour un module particulier ne se manifeste clairement dans les constructions gallo-romaines et la vérification mériterait d'être effectuée dans l'architecture de Rome. Pendant la Protohistoire, en France comme en Espagne, tout se passe comme si chaque agglomération avait élaboré ses propres modules. Comprenant souvent une brique et une demi-brique, ceux-ci variaient au cours du temps, comme on l'a démontré à Martigues et à Lattes (Chausserie-Laprée \& Nin 1987: 71; Chazelles 1997:60, fig. 52). On constate que le format théoriquement privilégié à l'époque augustéenne existe, parmi d'autres, à toutes les périodes ${ }^{17}$, mais on peut se demander si certaines dimensions courantes à partir des $\mathrm{II}^{\mathrm{e}}$ et $\mathrm{I}^{\mathrm{er}} \mathrm{s}$. av. $n$. è. autour de Marseille (Baou-Roux, Entremont, Glanum, Mimet), ainsi qu'à Lattes, ne s'insèrent pas, quand même, dans un système de briques et demi-briques, dont l'élément entier serait effectivement le module vitruvien. Par la suite, dans les villes romaines (Nimes, Orange, Aix-en-Provence, Lyon, etc), si les briques sont généralement rectangulaires, on n'enregistre pas non plus de format universel. 

ne pose pas de problème : les auteurs écartent les matériaux trop sableux ou trop riches en cailloux qui sont lourds et sujets à l'érosion. Plus difficile à comprendre, le terme sabulo, parfois traduit en français, à la suite de Claude Perrault, par «sablon mâle » et plus récemment par «sable grossier» (Croisille 1985: 274, note 17018), pourrait correspondre tout simplement à un matériau de texture limono-sableuse ou sablolimoneuse, c'est-à-dire à une terre fine, mais plus meuble que l'argile. L'énumération des matériaux recommandés par Vitruve sous-entend une réelle diversité granulométrique -depuis les argiles (plutôt que la craie retenue par certains traducteurs) et les ocres (terre rouge) à la texture très fine jusqu'à ce sabulo nécessairement plus grossier- qui correspond tout à fait à celle que l'on observe sur les adobes protohistoriques et romaines. Les constructeurs ont habituellement utilisé les matériaux disponibles dans leur environnement proche, quitte à les amender si leurs qualités plastiques laissaient à désirer: ajout de fibres végétales pour accroître la cohésion interne, inclusions minérales dans les terres trop argileuses, etc.

Mais que les terres soient blanches ou rouges n'a pas d'importance intrinsèque : ces couleurs évoquent soit des carrières connues de Vitruve autour de Rome, soit des terres qu'il a vu employer par des maçons. Bien souvent dans les ensembles bâtis, les briques montrent des teintes variées car les terres provenaient de carrières différentes ou de faciès distincts au sein d'une même carrière. Sur les sites de France méridionale et de Catalogne implantés dans des zones lagunaires ou des deltas de fleuves -tels que Lattes, Martigues, Salses, Montlaurès, Ullastret, etc.-, les couleurs des briques vont du jaune pâle au noir, selon que les limons sont issus de zones oxygénées par des apports réguliers d'eau claire ou de zones vaseuses, riches en matières organiques (photo 1).

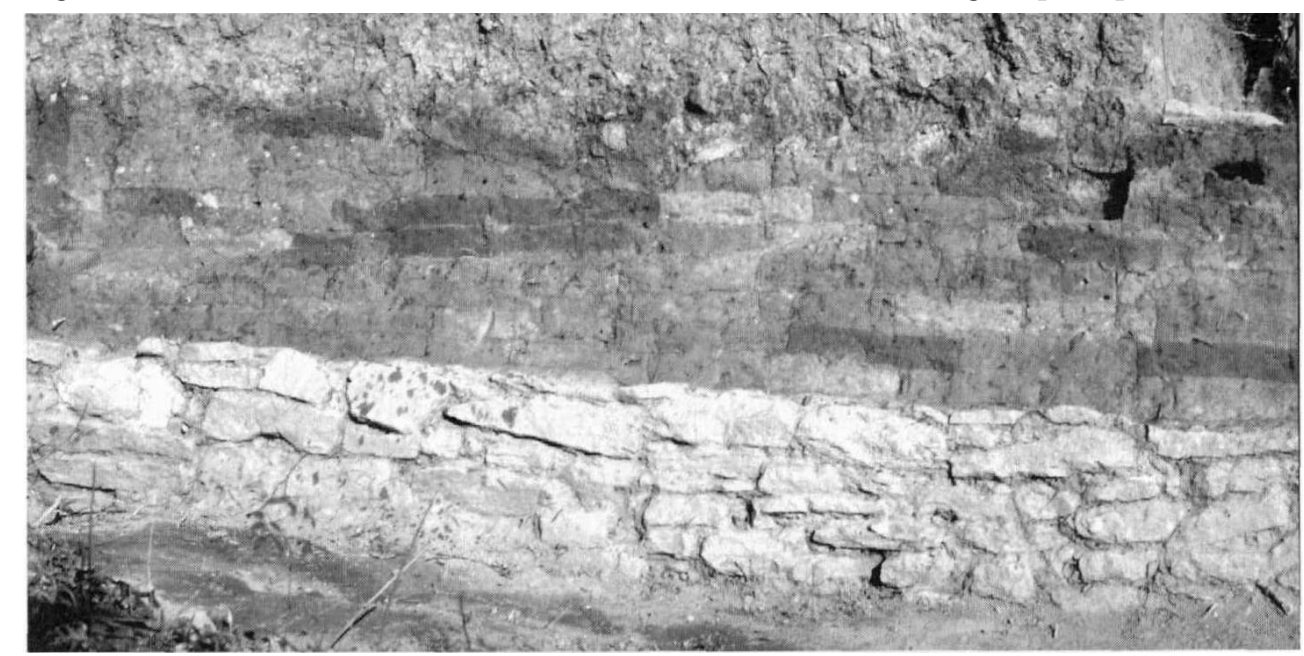

Photo 1. Mur en adobes de couleurs diverses sur soubassement en pierre. Habitat ibérique d'Illa d'en Reixac (Ullastret, Espagne). (H. Gazzal)

L'archéologie révèle aussi que les hommes n'ont pas toujours pris la peine d'aller extraire les matériaux idoines. Si cet effort se vérifie pour les périodes anciennes, aux $\mathrm{VI}^{\mathrm{e}}$ et $\mathrm{V}^{\mathrm{e}}$ siècles, à une époque où la brique représentait encore une technique nouvellement adoptée et pour laquelle les constructeurs ne s'étaient pas affranchis des normes qui l'accompagnaient ${ }^{19}$, il est rarement constaté pour les derniers siècles de l'Âge du fer. En effet, dans les agglomérations de Lattes, de Martigues ou du Marduel, les gravats produits par les démolitions de bâtiments ont fréquemment été substitués aux matériaux de carrières, afin de faire l'économie du transport. 

pailles correspond bien à une forme d'amendement des terres couramment enregistrée dans les briques gauloises et ibériques : les fibres végétales qui stabilisent la terre en limitant le retrait des argiles apparaissent sous la forme de négatifs quand on brise les adobes. Par ailleurs, l'expression macerari rencontrée chez Pline doit être comprise littéralement, plutôt que dans le sens d'« humecté » (Croisille 1985) : il est en effet recommandé par les constructeurs (contemporains) de laisser " pourrir » ou macérer pendant plusieurs jours la terre mélangée avec la paille et l'eau, afin que les mottes fondent, que les argiles aient le temps de gonfler et qu'une réaction chimique se produise sous l'effet de la fermentation des végétaux. Le mélange n'en sera que plus homogène et les risques de retrait diminués.

dans la manière d'agencer les briques que les témoignages archéologiques s'écartent le plus des textes latins. Aucun site gaulois ou ibérique n'a confirmé la disposition décrite par Vitruve, selon laquelle chaque assise de briques comporte une rangée de briques, accolée à une rangée de demi-briques : les parois mises au jour par les fouilles sont toujours constituées par une seule rangée de briques, le plus souvent disposées en panneresses (photos 1, 2 et 3). L'explication tient au fait que, pour Vitruve, les maisons romaines étaient destinées à posséder des étages, alors que les habitations de l'Âge $d u$ fer -et même celles du I ${ }^{\text {er }}$ siècle en règle générale- en comportaient rarement. Il est exact que la hauteur d'une paroi de brique est liée à son épaisseur ${ }^{20} \mathrm{et}$, comme le remarque l'architecte, on ne saurait élever un étage à partir de murs de largeur inférieure à un pied et demi. C'est d'ailleurs la raison pour laquelle les constructions en brique étaient proscrites à Rome.

essité d'élever les briques sur des soubassements en pierre pour limiter les remontées capillaires d'humidité dans les murs, prescrite par les auteurs latins (un pied de haut au minimum d'après Caton), n'avait pas échappé aux constructeurs protohistoriques car les murs périmétraux en sont toujours dotés, contrairement aux refends et aux cloisons qui reposent directement sur le sol (photos 1 à 3). Leur hauteur varie en fonction du contexte : terrain plus ou moins humide, sol plat ou en pente, etc., de la hauteur à donner aux édifices et de la disponibilité plus ou moins grande de pierres dans l'environnement.

37 Au registre de la protection des murs de brique crue, se trouve chez Vitruve comme chez Palladius une recommandation assez peu explicite, compte tenu de l'expression employée. Ils préconisent en effet de recouvrir les derniers centimètres des murs sous le toit d'une «structura testacea » formant une corniche saillante. Certains traducteurs, tel Pairault pour Vitruve, au XVII ${ }^{e}$ siècle, avaient opté pour " tuileau » qui désigne en réalité un mortier grossier de chaux et de céramique broyée ${ }^{21}$. Les traducteurs modernes considèrent qu'il s'agit simplement de briques cuites (comme René Martin dans sa traduction de Palladius ou L. Callebat pour le Livre II de Vitruve). Effectivement, dans d'autres chapitres du livre de Vitruve, par exemple quand il est question des maçonneries (II, 8 De generibus structurae), le mot testacea s'entend vraisemblablement comme un synonyme de brique cuite ou, à la rigueur, de tegula puisque l'on sait que les tuiles cassées étaient couramment remployées dans la maçonnerie des murs. On pourrait aussi imaginer qu'il s'agisse de pièces de terre cuite spécialement conçues, des sortes de larmiers servant à éloigner les eaux de ruissellement de la façade, mais on ne connaît pas de céramiques architecturales susceptibles d'avoir rempli cette fonction. Aussi la proposition de J.-M. Croisille semble- 
t-elle très satisfaisante car, selon lui, la structura testacea serait une maçonnerie formée par plusieurs rangs de briques cuites, disposés en surplomb, comme on voit de nos jours sur des maisons du sud-ouest de la France ${ }^{22}$. En tout cas, qu'il s'agisse de tuile ou de brique, il est peu probable que ce mode de protection ait eu cours en Gaule du temps de Vitruve car les Gaulois -qui n'avaient jamais employé la tuile comme matériau de couverture- ont tardé à adopter les terres cuites dans la maçonnerie. L'architecte en donne lui-même le témoignage en décrivant les toits en terre de Marseille ${ }^{23}$ ainsi que les couvertures en chaume ou en bardeaux de bois en usage dans plusieurs provinces.

La remarque de Vitruve au sujet de la forme des adobes, soulignant la préférence des Grecs pour la forme carrée, appelle quelques commentaires. En effet, alors que la plupart des gisements indigènes de Gaule ont produit uniquement des briques rectangulaires, certains ont eu également recours à des modèles carrés (photo 2). À Lattes, par exemple, des murs furent édifiés au IV siècle avec des briques qui s'apparentent au pentadorôn (environ $38-40 \mathrm{~cm}$ de côté), tandis que ces modèles ont été par la suite spécialement destinés à la réalisation de structures horizontales comme les sols ou les banquettes. Il est particulièrement significatif que dans la ville grecque d'Agde, le format de brique identifié soit non seulement carré à toutes les époques, mais qu'il corresponde de surcroît au pentadorôn grec évoqué par Vitruve ${ }^{24}$ (Nickels 1995 : 72). Par ailleurs, se pose la question de l'origine des briques crues, sur le plan général et plus particulièrement en Italie. Sous l'allusion légendaire qu'utilise Pline citant nommément deux "inventeurs " athéniens, transparaît incontestablement la connaissance de monuments bâtis en brique, très anciens et parfois réputés dans le monde grec. Vitruve, qui mentionne pour sa part, dans un autre chapitre, de nombreux édifices publics grecs comme des temples et des palais, ne dit rien au sujet de l'introduction de cette technique en Italie, en Gaule ou en Ibérie: cette manière de construire y est simplement constatée.

En Italie, les vestiges actuellement répertoriés par l'archéologie n'étant pas antérieurs à la colonisation grecque, on peut légitimement hésiter entre un réel apport hellénique, à l'instar de ce qui s'est produit en Gaule du sud, et une invention par les Etrusques puisque les plus anciens témoignages de briques crues -qui remontent au $\mathrm{VII}^{\mathrm{e}}$ sièclese rencontrent dans des agglomérations étrusques (Russellae ou Vetulonia, entre autres). On pourrait donc envisager une situation comparable à celle de l'Espagne où les peuples ibères du centre de la péninsule bâtissaient en briques moulées dès la fin de l'âge du bronze, dans des contextes culturels pré-coloniaux (Chazelles 1995). D'ailleurs, quoique Pline attribue à des Grecs l'invention de l'adobe, force est de constater, d'une part, l'usage traditionnel d'un format de brique rectangulaire en Italie qui se démarque des modèles helléniques et, d'autre part, le fait que cette brique est qualifiée de "lydienne » par le même Pline, comme par Vitruve. Or, on sait que cet adjectif était employé concurremment à celui d'étrusque par les Romains, en vertu de la provenance orientale de ce peuple, admise par plusieurs auteurs latins ${ }^{25}$ Il était donc reconnu implicitement, à l'époque d'Auguste, que le type de brique en vigueur avait pour sa part une origine strictement autochtone en Italie puisqu'il était « étrusque ».

En France, l'apparition de la brique moulée coïncide exactement avec l'arrivée des Phocéens à Marseille. En effet, quoique des fouilles récentes aient mis en évidence à Mailhac (Aude), dans un habitat du Bronze final IIIb (IX ${ }^{\mathrm{e}}-\mathrm{VIII}{ }^{\mathrm{e}}$ siècle av. $\mathrm{n}$. è.), des constructions formées par des blocs de terre plastique agglomérés, il s'agit là d'une variante de la bauge qui ne peut pas être retenue comme un antécédent gaulois de la 
brique proprement dite ${ }^{26}$. Les caractéristiques de la brique sont, d'une part, le moulage des éléments qui en facilite le calibrage et, d'autre part, le fait que ceux-ci sont préalablement séchés avant utilisation.

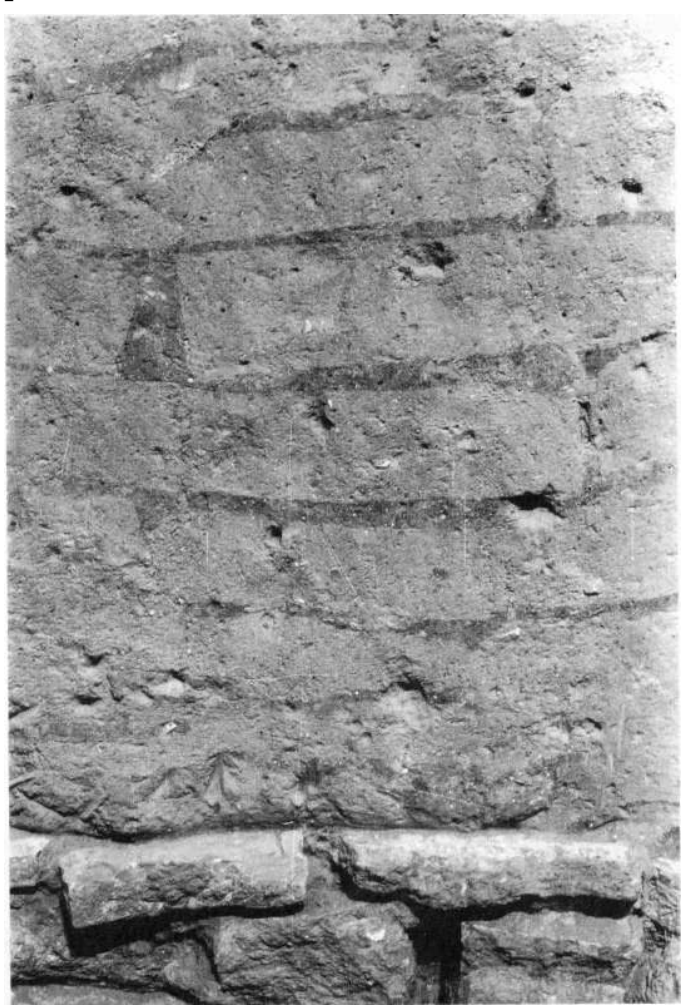

Photo 2. Mur en adobes rectangulaires, disposées en panneresses sur un solin en pierre. Habitat gaulois de Saint-Pierre-les-Marrigues (B. du Rh.). (C. Lagrand)

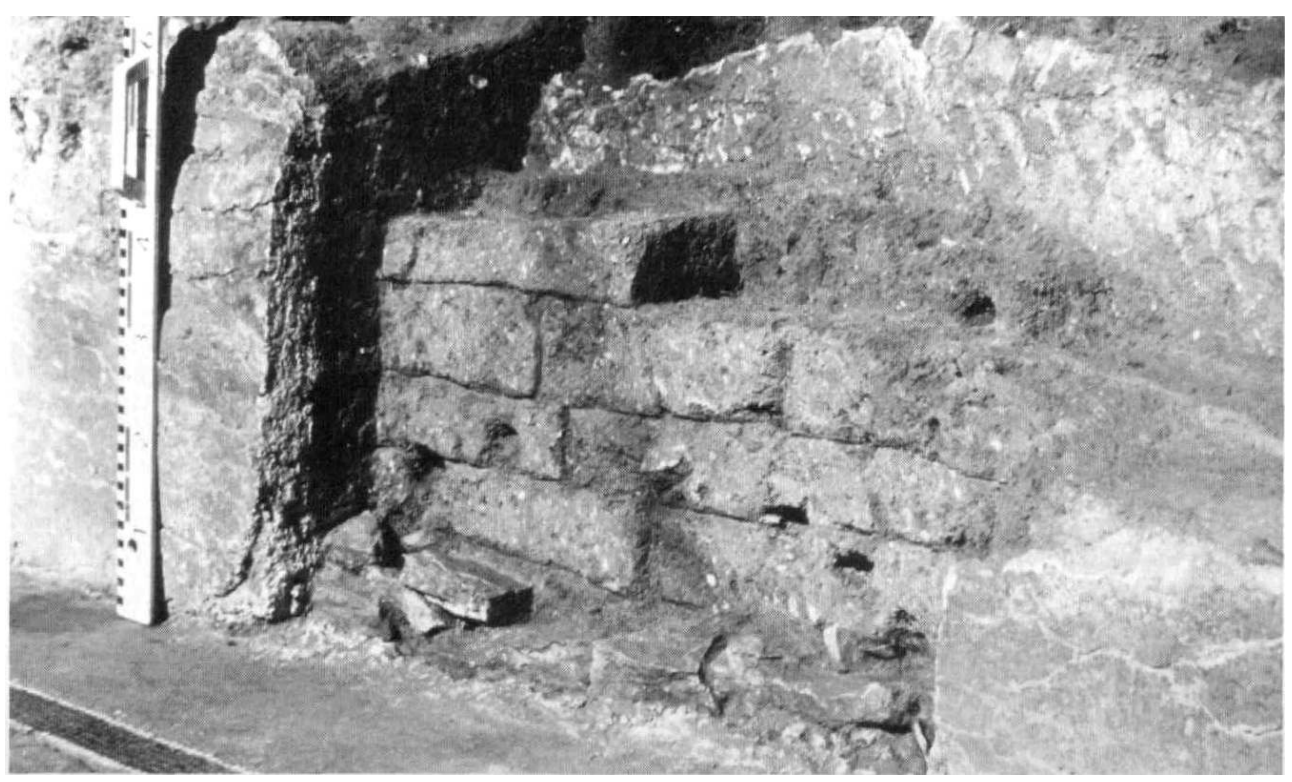

Photo 3. Mur de refend en adobe, sans soubassement. Habitat gallo-romain de Nimes (Gard). (P. Garmy) 


\section{La bauge ou terre massive} intérêt à ce mode de mise en œuvre de la terre crue, identifié jusqu'à maintenant uniquement dans des agglomérations gauloises employant conjointement la brique crue. Les gisements de Martigues (l'Ile et Saint-Pierre) où les maisons furent indifféremment bâties en terre massive ou en adobe entre la fin du $\mathrm{VI}^{\mathrm{e}} \mathrm{s}$. et le $\mathrm{I}^{\mathrm{er}}$ siècle av. n. è. (Chausserie-Laprée 2000; Chausserie-Laprée et Chazelles 2001), ainsi que plusieurs agglomérations voisines de l'étang de Berre attestant aussi l'usage de la bauge durant les $\mathrm{II}^{\mathrm{e}}$ et $\mathrm{I}^{\mathrm{er}}$ siècles av. n. è. (Marignane, Mouriès...), laissaient déjà pressentir le caractère autochtone de cette technique qui s'est maintenue en Provence durant tout l'Âge du fer, à côté de celle de la brique empruntée au monde grec ${ }^{28}$. En Languedoc, au contraire, ce procédé qui demande le maniement de très importants volumes de terre semble délaissé avant la fin du IV siècle C'est en tout cas ce que l'on observe à Lattes où les contraintes liées à l'urbanisme, à savoir l'insertion des habitations dans des lots aux limites strictes, ont pu conduire à préférer les murs de briques, plus étroits (Chazelles 1999). effectivement des largeurs imposantes, jamais inférieures à $60 \mathrm{~cm}$ et approchant plus souvent des $80 \mathrm{~cm}$, qui autorisent l'absence de solin en pierre, ces structures n'ayant pas à craindre la sape de leurs bases, contrairement aux parois de brique. Il est probable que leurs élévations présentaient un fruit important, ce que les vestiges conservés ne permettent pas d'appréhender (photo 4).

On constate une amélioration sensible de la technique sur les gisements provençaux, qui traduit une maitrise accrue des possibilités offertes par le matériau « terre » : au fil du temps les parois sont devenues plus étroites, se sont dotées de soubassements en pierre et ont certainement pu égaler les performances de la brique crue. La référence de Vitruve à des "mottes de terre" pourrait par conséquent s'appliquer à une des nombreuses variantes de la construction en bauge mises en évidence à la fouille (Chazelles 1999) et illustrées de nos jours par des comparaisons ethnographiques ${ }^{29}$. 


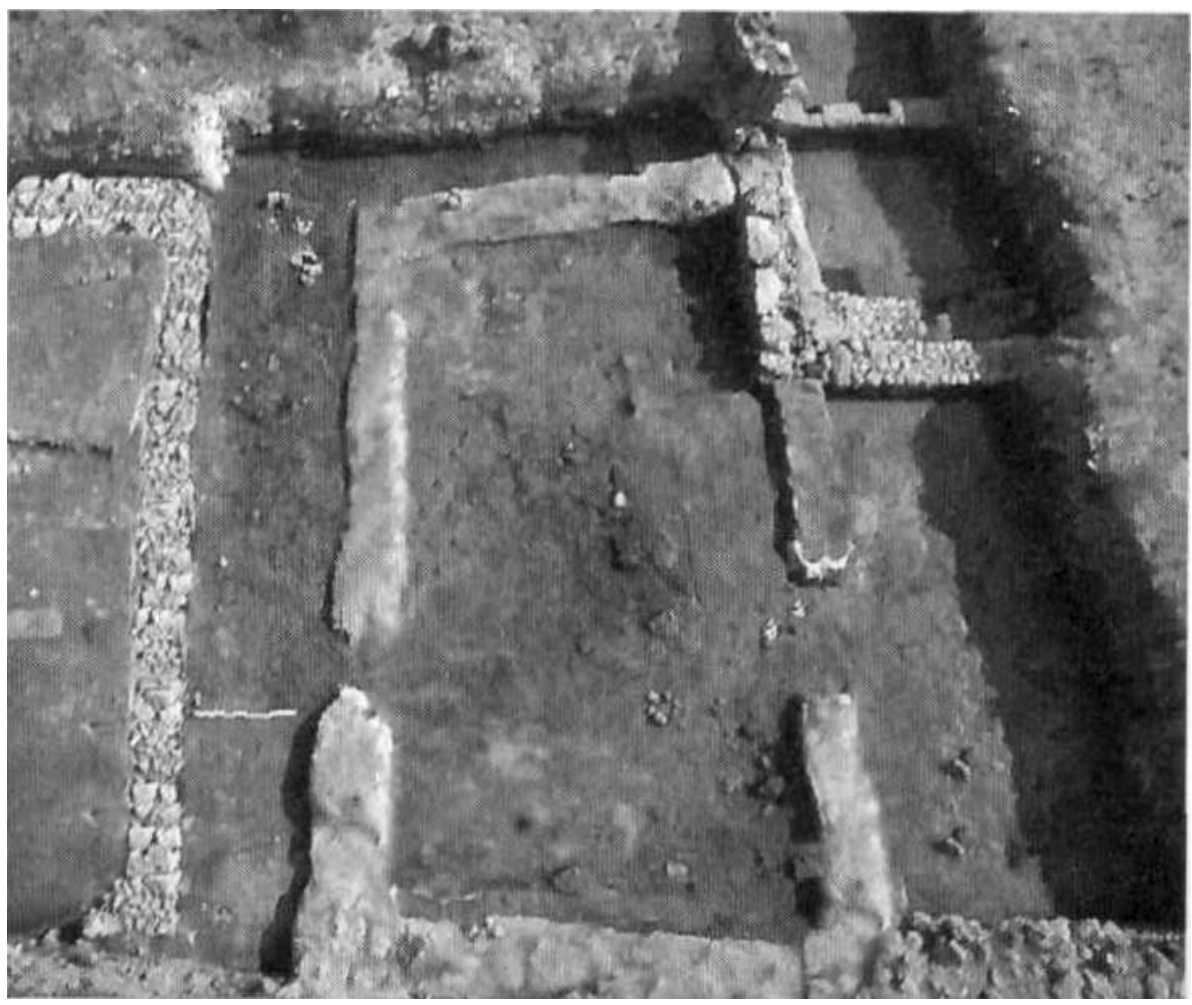

Photo 4. Habitation à murs de bauche. Agglomération gauloise de Lattes (Hérault). (J.-C. Roux)

\section{Le pisé}

"Pourquoi? est-ce qu'en Afrique et en Hispanie les murs de terre qu'ils appellent formaceos ("murs moulés") car ils sont bourrés dans un moule composé de deux planches placées de part et d'autre, plus qu'ils ne sont montés, n'affrontent-ils pas la durée, ne résistent pas aux pluies, aux vents, aux incendies plus solidement que n'importe quelle pierre? L'Espagne peut encore voir les postes d'observation d'Hannibal et ses tours de terre placées sur les chaînes de montagnes ${ }^{30}$.

51 Palladius: I, XXXIV, 4

52 (À propos des clôtures de jardin) «Certains, en enfermant de la boue entre des planches, imitent un peu les murs de terre $»^{31}$.

53 Trois remarques s'imposent: Vitruve ne dit pas un mot au sujet du pisé ; pour les agronomes, ce type de construction concerne en Italie uniquement des clôtures, jamais des murs de bâtiments ; enfin, ce type de structure si particulier évoque aussi bien pour Varron au début du I ${ }^{\mathrm{er}}$ siècle av. n. è. que pour Pline au troisième quart du Ier s. de n. è., des modèles connus en Espagne.

54 Les descriptions techniques données par Varron et Palladius ne laissent aucun doute sur la réalité qu'elles recouvrent : il s'agit bien de murs composés d'éléments moulés en place dans des « formes » ou des caissons en bois ${ }^{32}$, mais une différence notable entre 
leurs témoignages concerne le matériau constitutif. Selon Varron, les clôtures seraient faites avec un mélange de terre et de particules minérales tandis que Palladius se réfère, au contraire, à de la terre fine (luto évoquant de la boue). Il peut s'agir d'une interprétation personnelle de la part de ce dernier, s'il ne connaissait pas cette technique de visu, ou bien d'une variété de pisé propre à une zone particulière ${ }^{33}$.

Il apparaît, par conséquent, que la construction en pisé a été pratiquée en Italie avant le changement d'ère mais que tous les auteurs ne disposaient pas du même répertoire d'informations: en effet, ce genre de maçonnerie plutôt rustique a pu être mis en oeuvre uniquement dans certaines provinces italiques (provinces méridionales, peutêtre, puisque Tarente est citée), ce qui expliquerait l'ignorance de Vitruve. Quant à Pline, qui ne le mentionne pas en Italie non plus, il paraît l'avoir découvert en Afrique et en Espagne. Malgré la concision du texte, celui-ci atteste une connaissance directe du mode de fabrication et de l'extrême résistance des édifices en pisé que Pline avait dû constater par lui-même. Les détails de la mise en œuvre "entre deux planches latérales » et du " bourrage » dans le moule (inferciuntur) sont parfaitement clairs à cet égard.

Le témoignage de Pline est absolument essentiel pour l'histoire des techniques de construction. Sans chercher à gloser sur une tournure qui peut n'être que littéraire, il est quand même bien tentant de relever la formule «quos appelant » qui est une forme active. Il semble de la sorte que cette expression traduise un état de fait: ce sont les Hispaniques et les Africains qui nomment ainsi ce type de murs, pas les Romains. De plus, l'adjectif formaceus se présente de toute évidence comme un néologisme constitué par Pline à partir de forma (le moule, la forme) pour transcrire un terme inexistant en latin; en extrapolant à peine, on dira qu'avec cette expression il exprime dans sa langue une notion nouvelle. Si ces arguments linguistique et grammatical conservent une certaine fragilité, ils viennent quand même corroborer, à leur niveau, les sources archéologiques conduisant à attribuer aux peuples ibères et surtout puniques l'invention de la technique des murs banchés.

On ne connaît en effet aucun vestige de mur en terre damée dans les gisements gaulois du sud de la France, les seules structures en terre massive étant construites en bauge, comme on l'a vu précédemment. La question ne se résout pas si simplement en Espagne où, depuis le milieu du $\mathrm{XX}^{\mathrm{e}}$ siècle, les archéologues affirment découvrir du pisé dans l'habitat ibérique. Des mises au point, animées par la volonté de ré-examiner les documents sous l'éclairage apporté par les découvertes françaises de bauge, ont été faites récemment (Belarte 1997 ; Belarte \& Gailledrat 2001). Elles montrent qu'aucune certitude n'est acquise sut le sujet pour la période proprement dite ibérique $\left(\mathrm{V}^{\mathrm{e}}-\mathrm{II}{ }^{\mathrm{e}}\right.$ siècle av. n. è.). En revanche, une autre hypothèse est en train de voir le jour avec la possible mise en évidence de parois en terre tassée dans des niveaux du VII ${ }^{e}$ siècle av. n. è, sur un grand gisement phénicien de la côte andalouse (Guardamar del Segura) (Belarte \& Gailledrat 2001). C'est là une direction de recherche très séduisante puisque les seuls témoignages fiables antérieurs à notre ère dont on disposait jusqu'à présent étaient puniques. Sur les sites de Kerkouane, Carthage et Utique ont en effet été mises en évidence des constructions vraisemblablement en pisé damé, datant des $\mathrm{IV}^{\mathrm{e}}$ et $\mathrm{III}^{\mathrm{e}}$ siècles av. n. è. (Fantar 1984). Or, jusqu'à aujourd'hui, il n'était pas possible de préciser -faute de données sur les niveaux anciens des gisements d'Afrique- si les Phéniciens maîtrisaient déjà ce procédé à leur arrivée en Occident ou s'ils l'avaient mis au point au sein de leurs colonies : la datation haute des bâtiments de Guardamar pourrait fournir 
un élément de réponse en faveur de la première hypothèse. Il n'en demeure pas moins que les architectures ibériques, comme celles du Languedoc, ont toujours privilégié le recours à l'adobe et que les emplois présumés de pisé dans ce pays appartiennent à la fin de l'Age du fer (III ${ }^{\mathrm{e}} \mathrm{I}^{\mathrm{er}}$ siècles av. n. è.). L'agglomération d'Ampurias, dans le nord-est de la péninsule, a conservé plusieurs édifices de période romaine (deux domus et des boutiques autour du forum) assurément bâtis en pisé (photo 5). Datés du I ${ }^{\text {er }}$ siècle de $n$. è., les murs encore en élévation et protégés par des enduits peints sont formés par de la terre très sableuse qui conserve des signes évidents de coffrage ${ }^{34}$ (Chazelles 1990).

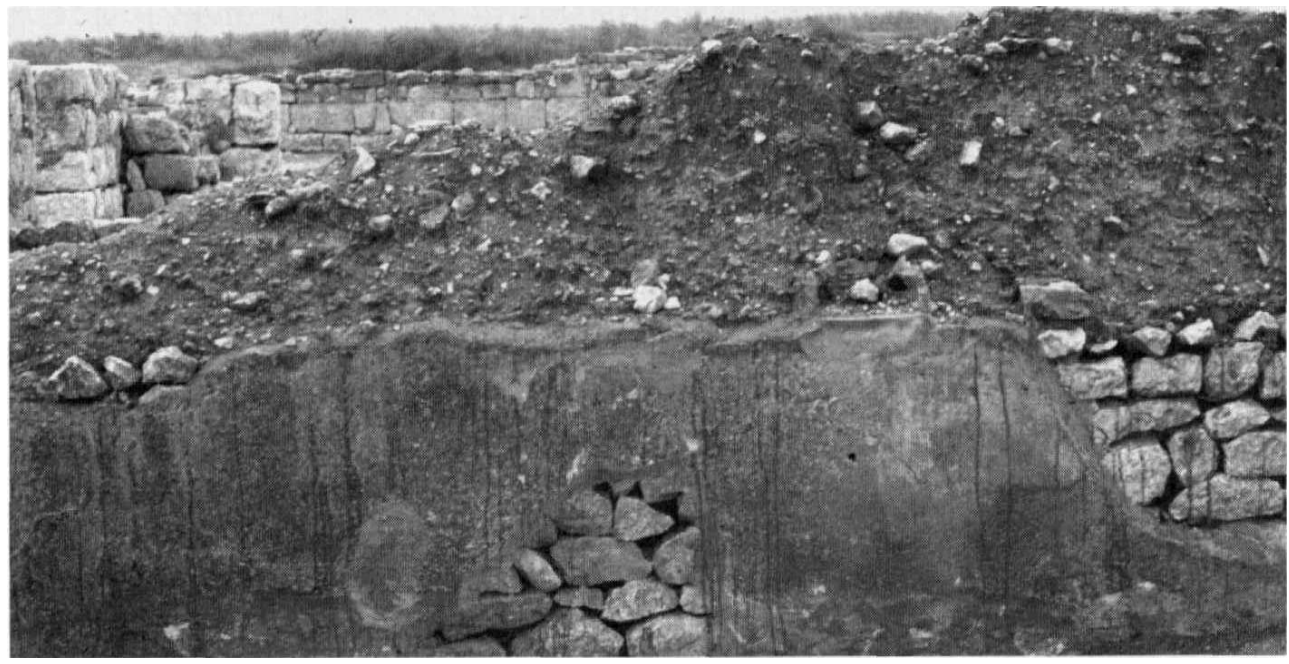

Photo 5. Mur en pisé sur solin en pierre. Habitat ibéro-romain d'Ampurias (Espagne). (C.-A. de Chazelles)

En France, aucun vestige d'époque protohistorique ne permet de conclure que les bâtisseurs pratiquaient cette technique et aucune démonstration n'a encore été faite non plus à propos de la construction gallo-romaine. Il est plus difficile de se faire une opinion au sujet des habitats des peuples italiques, car la recherche thématique sur l'architecture de terre crue est actuellement moins développée en Italie qu'en France ou en Espagne. La brève mention de Varron authentifie l'existence de structures en terre banchée et damée, dans le sud de la péninsule au cours du $\mathrm{I}^{\mathrm{er}}$ siècle av. n. è. Peutêtre est-ce sur la foi de ce témoignage que certains programmes de mise en valeur des sites n'ont pas hésité à reconstituer des maisons en pisé, alors même que les données archéologiques paraissent insuffisantes pour justifier ce choix ${ }^{35}$.

\section{Le pan de bois et le torchis}

Autant ils sont utiles pour gagner du temps et de l'espace, autant ils constituent une calamité commune et de tout premier ordre car ils sont comme des torches préparées pour les incendies [...]. De plus, sur les ouvrages crépis, des fissures se forment du fait de la disposition verticale et horizontale des montants. En effet, lorsqu'on les enduit, ils gonflent en s'imbibant d'humidité, puis se contractent en séchant et s'étant ainsi abîmés, ils nuisent à la solidité des enduits. [...], le soubassement sera assez haut pour éviter le contact avec le radier et le pavement ; car, s'ils [les éléments en bois] sont pris dedans, avec le temps, ils pourrissent $[. ..] »^{36}$. 
Vitruve, VII, 3

«[...] Mais, si des enduits doivent être posés sur des murs à clayonnages, il est inévitable que des fissures apparaissent le long des montants verticaux et horizontaux, parce que quand on les recouvre de terre, ils s'imbibent forcément d'humidité, puis quand ils sèchent ils se rétractent et provoquent des fissures dans les enduits, pour éviter cela... [...] $»^{37}$

Pline XXXV, 48, 14

"Qui ignore en effet que les murs faits de clayonnages sont enduits de terre et qu'ils sont dressés à la manière des briques crues ? ${ }^{38}$

Palladius I, 19, 2

(À propos de la construction des greniers) « [...] il suffira ou bien de diviser les greniers au moyen de séparations en claies $[. ..] »^{39}$.

Le principe consistant à enduire des claies (ou clayonnages) de branchages avec de la terre, que l'on désigne en français par le mot «torchis », faisait partie des procédés de construction employés par les Romains, même si Vitruve le rejette en raison de sa vulnérabilité au feu. Mais on ne peut pas donner aux parois faites de crates le sens restrictif de constructions en torchis car, de toute évidence, l'expression s'applique à des structures plus complexes, c'est-à-dire à des pans de bois hourdés de torchis. Le fait que Palladius admette ce procédé pour diviser l'espace des greniers évoque des séparations légères, basses et sans rôle porteur. En revanche, les deux extraits du livre de Vitruve comportent assez d'indices révélant des constructions à charpentes de bois, dans lesquelles les «claies" interviennent simplement en guise de remplissage. De plus, les mises en garde concernant les risques de pourriture du bois, si les sablières basses sont prises dans les pavements et ne reposent pas sur des solins maçonnés, confirment qu'il s'agit de murs de rez-de-chaussée, peut-être même de murs extérieurs. Il est intéressant de relever, cependant, que ces murs peuvent éventuellement être laissés sans enduits.

Pour traiter rapidement l'historique du torchis, disons que ce procédé est un des plus anciens que l'homme ait inventé pour édifier l'habitat de plein air, comme l'attestent d'innombrables exemples qui remontent au Néolithique, sur le pourtour de la Méditerranée ainsi qu'en Europe tempérée. Mais s'il est resté en usage jusqu'à l'époque romaine dans une bonne partie des pays européens, le torchis en tant que tel a pratiquement disparu des architectures méditerranéennes, laissant la place à la pierre et à la brique crue, plus rarement à la bauge ${ }^{40}$.

En Gaule, la construction à pans de bois (ou à colombages) n'est apparue qu'à l'extrême fin de l'Âge du fer (à Entremont, fin II $^{\mathrm{e}}$ ou début $\mathrm{I}^{\mathrm{er}}$ siècle av. n. è.) et s'est généralisée à l'époque gallo-romaine. Si elle ne semble pas attestée en Ibérie, sauf peut-être dans les régions de piémont des Pyrénées, elle est en revanche mise en évidence en Italie durant tout l'Âge du fer et sous l'empire romain. Dans certains habitats étrusques, notamment à Marzabotto dès la fin du VI ${ }^{\mathrm{e}}$ siècle, ainsi qu'à Acquarossa, les charpentes de bois étaient hourdées de torchis (Massa-Pairault 1997: 93, note 30 et fig. 20). Pour des périodes plus récentes, des hourdis en adobe ont été signalés à Gravina, par exemple, dans des maisons du $\mathrm{II}^{\mathrm{e}}$ siècle av. n. è. (Small 1992 : 16). Enfin, les maisons urbaines de Pompei et d'Herculanum montrent la banalisation des pans de bois, au $\mathrm{I}^{\mathrm{er}} \mathrm{s}$. de n. è., à travers les étages construits en encorbellement sur les rues. 
70 Vitruve et, pour ce qui est de la France, ils révèlent que la technique du pan de bois a vraisemblablement été introduite par les Romains, au même titre que les mortiers à base de chaux et les diverses terres cuites architecturales. Les attestations de murs porteurs à colombages, avec sablière continue ou discontinue, isolés du sol par des solins en pierre, sont en effet assez nombreuses sous le Haut Empire : Narbonne, Nimes, Lunel-Viel, Sallèles d'Aude, Aix-en-Provence, Olbia, Lyon, etc. D'autres exemples montrent des cloisons élevées directement à partir du sol sur des sablières basses (photo 6). La préférence a souvent été donnée aux hourdis de briques crues, qui peuvent être disposées sur chant, en particulier pour les cloisons étroites (Narbonne, Nimes, Lyon), mais le torchis sur clayonnage n'a pas été négligé, surtout dans les régions septentrionales où cette technique s'était perpétuée depuis le Néolithique (Normandie, Lorraine, région Lyonnaise ${ }^{41}$ ).

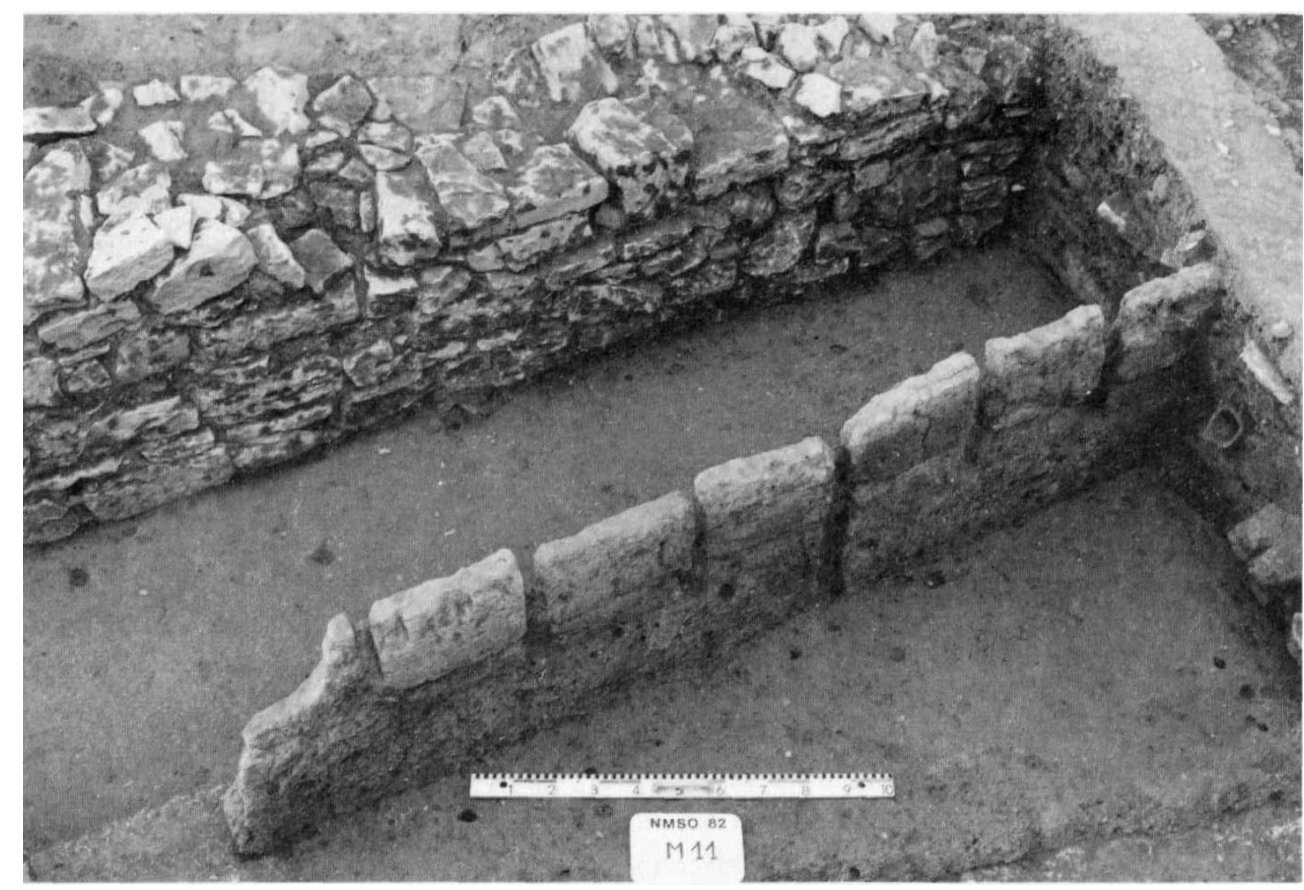

Photo 6. Cloison à pan de bois hourdé d'adobes de chant. Habitat gallo-romain de Nimes (Gard) (P. Garmy)

En dépit de leur caractère elliptique, les références latines sur l'architecture domestique s'avèrent d'une fiabilité inattendue. Chaque fois que des fouilles archéologiques ont été en mesure de le vérifier grâce à une bonne conservation des vestiges, on a pu constater une adéquation entre les descriptions techniques ou les témoignages ethnographiques et les constructions elles-mêmes. Il faut cependant se garder d'une foi aveugle quand on veut utiliser ces sources pour écrire ou compléter l'histoire des techniques de construction, car le véritable problème ne tient pas à la véracité des observations mais à leur manque d'exhaustivité. Par exemple, le fait que Vitruve ne dise mot au sujet des structures en pisé ou qu'il ne mentionne pas les hourdis de briques crues dans les murs à pans de bois ne signifie pas que les premières lui étaient inconnues ni que les seconds n'avaient pas cours à Rome. Les murs de clôture en pisé de la région de Tarente ont pu lui sembler sans intérêt pour un ouvrage didactique et sa diatribe sur les risques d'incendie que comportent les murs à ossature 
de bois aurait été moins pertinente s'il n'avait pas comparé les clayonnages à des fagots prêts pour le feu.

in traité dans cet article que la question des murs, mais les rapprochements entre les sources écrites et les témoins archéologiques se montrent également probants dans d'autres domaines de la construction, comme ceux des toitures, des plafonds ou des enduits muraux. Avoir pu vérifier l'exactitude des témoignages antiques sur quelques points précis permet d'accréditer d'autres assertions plus difficiles à contrôler... Par exemple en ce qui concerne les ajouts de matières organiques souvent signalés par les agronomes et, spécialement, l'amurca ce résidu liquide du pressage des olives, car l'ethnographie révèle le rôle important que joue, dans la cohésion des terres à bâtir, l'adjonction de déjections animales, de sucs végétaux divers, voire de matières organiques fossiles.

De fait, la valeur essentielle de ces textes ne réside pas dans les informations techniques, que l'on peut en grande partie acquérir par les recherches de terrain, mais plutôt dans la présentation des manières d'habiter et de construire propres à chaque culture, région ou pays dont ils témoignent.

\section{BIBLIOGRAPHIE}

Baudreu, Dominique

2001, « Habitats et fortifications en terre crue d'époque médiévale, dans le Midi de la France », Communication orale à la table ronde «Échanges transdisciplinaires sur les constructions en terre crue. I. Les techniques de la terre massive. Montpellier, 17-18 novembre.

Belarte, Maria-Carme

1997, « Arquitectura domèstica i estructura social a la Catalunya protohistòrica », Arqueo Mediterrània 1.

Belarte, Maria-Carme \& Eric Gailledrat 2001, « Murs protohistoriques de terre massive sur la côte orientale de la péninsule ibérique (VII ${ }^{\mathrm{e}}-\mathrm{III}{ }^{\mathrm{e}}$ s. av. J.-C). L'exemple de Guardamer del Segura (province d'Alicante) », Communication orale dans : Échanges transdisciplinaires sur les constructions en terre crue. I. Les techniques de la terre massive, table-ronde, Montpellier, 17-18 novembre.

Caton

1975, De Agricultura, (édité par R. Goujard). Paris : Les Belles Lettres (Collection des Universités de France).

Chausserie-Laprée, Jean 2000, «Villages gaulois en Provence : genèse et évolution du fait urbain », pp. 31-50, Le temps des Gaulois en Provence. Ville de Martigues/Musée Ziem.

Chausserie-Laprée, Jean \& Nuria Nin 1987, « Le village protohistorique de l'Ile à Martigues (II) », Documents d'Archéologie Méridionale 10 : $31-90$. 
Chausserie-Laprée Jean \& Claire-Anne Chazelles (de)

2001, « La terre massive façonnée, un mode de construction indigène en Gaule du Sud, et la question du pisé dans l'Antiquité ", Communication orale à la table ronde Échanges transdisciplinaires sur les constructions en terre crue. I. Les techniques de la terre massive. Montpellier, 17-18 novembre.

Chazelles, Claire-Anne (de)

1990, «Les constructions en terre crue d'Empuries à l'époque romaine », Cypsela Numéro VIII :

101-118.

1995, «Les origines de la construction en adobe en Extrême-Occident ", Études Massaliètes 4 :

49-58.

1996, "Les techniques de construction de l'habitat antique de Lattes ", Lattara $9: 259-328$.

1997, Les maisons en terre de la Gaule méridionale. Monographies Instrumentum 2. Montagnac:

Éditions Monique Mergoil.

1999, « À propos des murs en bauge de Lattes : problématique des murs en terre massive dans

l'antiquité », Lattara $12: 229-254$.

Chazelles, Claire-Anne (de) \& Pierre Poupet

1984, «L'emploi de la terre crue dans l'habitat gallo-romain en milieu urbain : Nimes », Revue

Archéologique de Narbonnaise XVII : 71-101.

Coarelli, Filippo

1973, Les cités étrusques. Paris : Éditions Bordas.

Columelle

1845, De Re rustica (traduction L. du Bois). Paris : Éditions Panckoucke. Desbat, Armand 1981, « L'architecture de terre à Lyon à l'époque romaine », BAR, International series, 108 : 55-81. 1985, « La région de Lyon et de Vienne », Documents d'Archéologie Française 2 (« Architectures de terre et de bois ») : 75-83.

1987, « La construction en terre à l'époque romaine ", Le patrimoine européen construit en terre et sa réhabilitation, colloque international ENTPE, 18-20 mars 1987, p. 469-487 (pré-actes, document polycopié).

Dion Cassius

1870, Histoire romaine. Paris : Librairie Firmin Didot.

Doat, Patrice, Hays Alain, Houben Hugo, Matuk Silvia et François Vitoux 1979, Construire en terre. Paris : Éditions Alternative et Parallèles (« Anarchitecture »).

Documents d'Archéologie Française 2

1985, Architectures de terre et de bois. L'habitat privé des provinces occidentales du monde romain.

Antécédents et prolongements : Protohistoire, Moyen Age et quelques expériences contemporaines. Paris : Éditions de la Maison des Sciences de l'Homme.

Fantar, Mohamed

1984, Kerkouane, cité punique du Cap Bon (Tunisie). I. Tunis. : Institut National d'Archéologie et d'Art.

Greco, Giovanna

1991, Serra di Vaglio, La casa dei pithoi. Modena : F.-C. Panini éditeur.

Jallot, Luc

2001, «Les constructions en terre massive et en blocs façonnés du Néolithique et du Chalcolithique dans le Sud de la France », Communication orale à la table ronde Échanges transdisciplinaires sur les constructions en terre crue. I. Les techniques de la terre massive. Montpellier, 17-18 novembre. 
Klein, Alain

2001, « Le patrimoine architectural en terre crue de Midi-Pyrénées (XV - XX Communication orale à la table ronde Échanges transdisciplinaires sur les constructions en terre crue. 1. Les techniques de la terre massive. Montpellier, 17-18 novembre.

Lequément, Robert

1985, « L'apport des textes antiques », Documents d'Archéologie Française 2 (« Architectures de terre et de bois ») : 29-32.

Massa-Pairault, Françoise-Hélène (dir.)

1997, Marzabotto. Recherches sur l'insula V, 3. Rome : Collection de l'École française de Rome, 228.

Nickels, André

1995, « Les sondages de la rue Perben à Agde (Hérault) », Études massaliètes 4 : 59-98.

Palladius

1976, Opus agriculturae, livres I et II (édité par R. Martin), Les Belles Lettres, Collection des Universités de France. Paris.

Pline l'ancien

1977, Naturae Historiarum, Livre VII, (édité par R. Schilling). Paris : Les Belles Lettres (Collection des Universités de France).

1985, Naturae Historiarum, Livre XXXV (édité par J.-M. Croisille). Paris : Les Belles Lettres

(Collection des Universités de France).

Small, Alistair, M.

1992 « Gravina. An Iron Age and republican settlement in Apulia », Archœological Monographs of the British School at Rome, 5.

Scherrer, Olivier

2001, « Actualité de la construction en bauge en Afganistan : la technique du "pakhsa" "

Communication orale à la table ronde Échanges transdisciplinaires sur les constructions en terre crue.

I. Les techniques de la terre massive. Montpellier, 17-18 novembre.

Seignobos, Christian

1982, Nord Cameroun, Montagnes et hautes terres. Roquevaire : Éditions Parenthèses (« Architectures traditionnnelles »).

Strabon

1960, Géographie, XIII (ed. by T.E. Page et al.), english translation by H.-L. Jones. Londres : Harvard University Press (The loeb Classical Library).

1966, Géographie, III et IV (Éditions Lassere). Paris : Les Belles Lettres (Collection des Universités de France).

Suetone

1931, Vies des douze césars, t. 1, Auguste, (édité par H. Ailloud). Paris : Les Belles Lettres

(Collection des Universités de France).

Varron

1978, Res rusticœ, Livre I, (éditeur J. Heurgon). Paris : Les Belles Lettres (Collection des Universités de France).

Vitruve

1979, Les dix livres d'architecture (traduction de Claude Perrault, 1673). Balland.

1995, De Architectura, livre II (édité par B. Liou, M. Zuinghedau, M.-T. Cam), Les Belles Lettres, Collection des Universités de France, Paris. 
1999, De Architectura, livre II (édité par L. Callebat). Paris : Les Belles Lettres (Collection des Universités de France).

Wattez, Julia

2001, « Caractérisation micromorphologique des matériaux façonnés en terre crue dans les habitats néolithiques du sud de la France : l'exemple des sites de Jacques Cœur (Montpellier, Hérault), du Jas del Biau (Millau, Aveyron) et de La Capoulière (Mauguio, Hérault) », Communication orale à la table ronde Échanges transdisciplinaires sur les constructions en terre crue. I. Les techniques de la terre massive. Montpellier, 17-18 novembre.

\section{NOTES}

2. Voir par exemple le congrès sur « les architectures de terre et de bois » qui s'est tenu à Lyon en 1983 (Documents d'Archéologie Française 1985).

3. Strabon, Géographie, IV, 4, 3 et XIII, I, 67.

4. Dion Cassius, Histoire romaine, XXIX, 61.

5. Suetone, Vies des douze césars, Auguste, 28.

6. [...] lapide calce fundamenta supra terram pede, ceteros parietes ex latere.

7. Huius fere species quattuor, quod fiunt e lapide, ut in agro Tusculano, quod e lateribus coctilibus, ut in agro Gallico, quod e lateribus crudis, ut in agro Sabino, quod ex terra et lapillis compositis in formis, ut in Hispania et agro Tarentino.

8. Non enim de harenoso neque calculoso neque sabulonoso luto sunt ducendi, quod, ex hisgeneribus cum sint ducti, primum fiunt graves, deide, cum ab imbribus in parietibus sparguntur, dilabuntur et dissoluntur paleaque in his non cohaerescunt propter asperitam. Faciendi autem sunt ex terra albida cretosa sive de rubrica aut etiam masculo sabulone.

9. Fiunt autem laterum genera tria: unum, quod graece Lydium appellatur, id est quo nostri utuntur, lungum sesquipede, latum pede. Ceteris duobus Graecorum aedificia struntur: ex his unum pentadoron alterum tetradoron dicitur. [...] Fiunt autem cum his lateribus semiletaria. Quae cum stuuntur una parte lateribus ordines, altera semilaleres ponuntur.

10. Latericii vero, nisi diplinthii aut triplinlhii fuerint, sesquipedali crassitudine non possunt plus unam sustinere contignationem.

11. Summis parietibus testacea sub tegula subiciatur altitudine circiter sesquipedali habeatque projecturas coronarum.

12. Lateres non sunt ex sabuloso neque harenoso multoque minus calculoso ducendisob, sed ecreteso et albicante aut ex rubrica uel etiam e sabuloso, masculo certe.[...] et intritam ipsam eorum, priusquam fingantur, macerari oportet. Genera eorum, quae tria, Lydion quo utimur, longum sesquipedem, latum pedem [...].

13. Laterarias ac domus constituerunt primi Eutyalous et Hyperbius frates Athenis [...]

14. Quod si latericios parietes in praetorio facere volveris, illud servare debebis, ut perfectis parietious in summitate, quae trabibus subiacebit, structura testacea cum coronis prominenlibus fiat sesquipedali altitudine [...].

15. Sint vero lateres longitudine pedum duorum, latitudine unius, altitudine quater unciarum.

16. Néanmoins ce format est notamment attesté dans une construction nimoise du $\mathrm{I}^{\mathrm{er}} \mathrm{s}$. de $\mathrm{n}$. è. $(\geq 60 \times 36 \times 7 \mathrm{~cm})$ (Chazelles \& Poupet 1984).

17. En effet, si ce module a bien été employé à Narbonne et à Olbia, à l'époque augustéenne, il existe aussi sur des sites protohistoriques beaucoup plus anciens (comme Montlaurès ou Martigues, par exemple, dès le $\mathrm{V}^{\mathrm{e}} \mathrm{s}$. av. J.-C).

18. «Il s'agit d'un sable grossier qui ne pouvait être qu'un ingrédient de fabrication ». 
19. Sur le site de hauteur du Marduel par exemple, les briques anciennes ont été moulées à partir de terre extraite à plusieurs kilomètres et qu'il a fallu transporter (Chazelles et al. 1985 : 69).

20. On admet que la hauteur d'un mur en brique peut atteindre huit fois sa largeur (Doat et al. $1979: 121)$.

21. Voir par exemple le chapitre sur les sols : pavimenta, Vitruve, VII, 1.

22. Un procédé similaire, mais transposé puisqu'il est réalisé à l'aide de tuiles courbes (imbrices), se rencontre également en Italie et en France méridionale où il prend le nom de " génoise ». Son apparition date peut-être du Moyen-Âge, lorsque les tuiles plates ont complètement disparu des toitures.

23. Vitruve II, 1 : «À Marseille, au lieu de tuiles, les maisons sont couvertes de terre pétrie avec de la paille $»$.

24. À Marseille en revanche, les briques répertoriées sont le plus souvent franchement rectangulaires, bien que certaines se rapprochent du carré (par exemple: $32 \times 28 \mathrm{~cm}$ ou $21 \times 17 \mathrm{~cm}$ ).

25. Hérodote, notamment, reconnaissait dans les Etrusques les descendants démigrants originaires de Lydie, en Asie Mineure (Hérodote, Histoires, I, 94).

26. Ce mode de construction a été mis en évidence à Mailhac, sur le gisement de plaine du Traversant, par E. Gailledrat (com. pers.).

27. Primumque furcis erectis et virgulisinterpositis luto parietes texerunt. Alii luteas glaebas arefacientes struebant parietes [...].

28. Le caractère indigène et très ancien des techniques du façonnage direct est désormais démontré par les travaux des préhistoriens du Languedoc. Plusieurs gisements de plaine datés du Néolithique final et du Chalcolithique ont livré au cours des dernières années des structures bâties à l'aide de mottes et/ou de blocs de terre découpés (Jallot 2001 ; Wattez 2001). Toutefois, compte tenu de la durée qui sépare les habitats chalcolithiques et Bronze final -plus d'un millénaire- la pérennité des techniques constructives ne peut pas être établie dans la région.

29. En Afghanistan ou au Yémen, on construit des murs épais à partir de boules de terre humide projetées (Scherrer 2001), tandis que dans certains pays d'Afrique noire cette technique s'adapte à des parois très étroites (au Nord Cameroun: Seignobos 1982). D'autres habitats africains montrent une préférence pour des mottes partiellement séchées ou des blocs découpés (Guillaud 2001). La même distinction a été observée sur des bâtiments médiévaux et sub-contemporains du sud-ouest du Midi de la France (Baudreu 2001 ; Klein 2001).

30. Quid? Non in Africa Hispaniaque e terra parietes, quos appelant formaceos, quoniam in forma circumdatis II utrimque tabulis inferciuntur verius quam struuntur, aevis durant, incorrupti imbribus, ventis, ignibus

31. Alii luto inter formas clauso parietes figulatos ex partibus imitantur.

32. Les restitutions de la fin de la phrase varient selon les éditeurs du texte latin. Pour R. Martin (Belles Lettres, 1976), il faut renoncer à figuratos ex lateribus donné par ses prédécesseurs, au profit de figulatos ex partibus. Dans ce dernier cas, il n'est plus question de ressemblance avec une maçonnerie « de brique » mais d'une simple comparaison avec les murs « de terre ».

33. Dans certaines régions de France, en particulier dans la vallée de la Durance, on peut encore voir des murs en pisé formés par des limons alluvionnaires très fins, peu adaptés au compactage et qui ont d'ailleurs tendance à se fissurer.

34. Il convient dès lors de poser clairement la question au sujet des structures en terre massive repérées sur quelques gisements de la période ibérique récente (III ${ }^{\mathrm{e}}-\mathrm{II}^{\mathrm{e}}$ siècles) : a-t-on affaire, comme en France méridionale, à la perpétuation de la tradition de la bauge (que l'on commence aussi à mettre en évidence dans des contextes plus anciens) ou bien, déjà, à du pisé coffré ? Dans ce cas, l'allusion de Pline à des tours de guet en terre qu'il attribue à Hannibal tendrait à accréditer la théorie d'une influence punique en Espagne. 
35. Sur le site de Serra de Vaglio (Basilicate), la maison dite «Casa dei pithoi », datée du IV siècle av. n. è. a été ainsi restituée entièrement en pisé. Les arguments qui fondent le choix de cette technique plutôt que celui de l'adobe ou du torchis, n'apparaissent pas déterminants dans l'article consacré à la restitution (Greco 1991). On invoque des fragments composés d'argile et de paille qui comportent «la caratteristica traccia delle casseformi di legno » (ibid., 61), mais qui pourraient peut-être correspondre à des hourdis de torchis entre des pans de bois.

36. Craticii vero velini quidem ne inventi essent. Quantum enim celeritate et loci laxamento prosunt, tanto maiori et communi sunt calamiti quod ad incendia uti faces sunt parati [...] Etiamque in tectoriis operibus rimas in his faciunt arrectariorum et transversiorum dispositione. Cum enim linuntur, recipientes umorem turgescunt, deinde siccescendo contrahuntur et ita extenuati disrumpunt tectoriorum soliditatem. [...] Solum substruatur alte, ut sit intactum dondere et pavimento; obruta enim in bis cum sunt, vetustate marcida fiunt [...].

37. Sin autem in craticiis tectoría erunt facienda, quibus necesse est in arrectariis et transversariis rimas fieri, ideo quod, luto cum linuntur, necessario recipiunt umorem, cum autem arescunt, exlenuati in tectoriis faciunt rimas, id ut non fiat [...].

38. Inlini quidem crates parietum luto et lateribus crudis exstrui quis ignorat? Je ne suis pas d'accord avec la restitution du texte donnée par J. Croisille », $1985: 109$ et note 169, 5) : « On les édifie ainsi en espèces de briques crues car on ne peut pas admettre de ressemblance entre les murs de torchis et les constructions appareillées en briques, ni visuellement sur le plan formel, ni même en considérant avec lui qu'il y a une assimilation entre le matériau (terre et paille) et les deux modes de mise en œuvre». La phrase, qui fait suite au paragraphe sur le pisé, renvoie simplement à deux autres procédés utilisant aussi la terre crue.

39. [...] vel craticiis podiis erunt discernenda granaria.

40. Dans le Midi de la France, on le rencontre le plus souvent dans la réalisation de cloisonnements internes ou de parois de bâtiments annexes. Les murs périphériques en torchis sur poteaux porteurs restent des exceptions et l'on ne mentionnera qu'un exemple, daté du IV siècle, à Lattes (Roux \& Chabal 1996).

41. Sur le rôle de la terre crue dans la construction lyonnaise sous le haut empire, voir les travaux pionniers de A. Desbat $(1981,1985,1987)$.

\section{RÉSUMÉS}

Appliquée au domaine de la construction en terre crue, la mise en parallèle de quelques sources latines et de vestiges archéologiques a permis de vérifier la validité des premières et de conforter certaines hypothèses relatives à l'histoire des techniques. L'article se limite à l'examen des données concernant les murs, les plus riches des points de vue textuel et archéologique. Les citations, écrites entre le II ${ }^{e}$ siècle av. n. è. et le IV siècle de n. è., sont respectivement extraites du traité d'architecture de Vitruve, de l'Histoire naturelle de Pline, ainsi que des ouvrages des agronomes Varron, Caton et Palladius. Quant aux vestiges de constructions domestiques, ils datent pour partie de l'Âge du fer (VI $\mathrm{e}^{\mathrm{e}} \mathrm{I}^{\mathrm{er}}$ s. av. n. è.) et pour partie de la période romaine. La plupart sont issus de gisements de la France méridionale et de la Catalogne espagnole.

A comparison of Latin literature and archaelogical remains of mud walls has established the validity of the former as well as reinforcing certain hypotheses about the history of techniques. The article examines data concerning walls, using a great deal of information as far as both the texts and the archacology go. The quotations used, written between the $2^{\text {nd }}$ C. B.C. and $4^{\text {th }}$ C. A.D 
have been taken from the treatise on architecture by Vitruvius and Pliny's Natural History, as well as works by Varron, Cato, Columelle and Palladius. The homes whose walls were examined date from the Iron Age ( $6^{\text {th }}-1^{\text {st }}$ C. B.C.), and the Roman period, most of them in settlements from Southern France or from Spanish Catalonia.

La confrontación de algunas fuentes latinas y de unos vestigios arqueológicos permite averiguar la validez de aquellas y precisar ciertas hipótesis acerca de la historia de las técnicas de construcción en tierra cruda. Este artículo examina solo los datos que respectan a los muros, ya, que entregan la mejor información arqueológica y textual.

INDEX

Mots-clés : constructions antiques, terre crue, textes latins, architecture

\section{AUTEUR}

\section{CLAIRE-ANNE DE CHAZELLES}

UMR 154, CNRS, 390 avenue de Pérols, 34970 Lattes 